Series A

I. MATHEMATICA

333

\title{
ON THE COMPLETION OF UNIFORM SPACES
}

BY

TOIVO NIEMINEN

H E L S I K I 1963

S U O M A L A I N E T T E D E A A T E M A 
Communicated 8 March 1963 by P. J. Myrberg and Olli Lehto

HELSINKI 1963 


\section{On the completion of uniform spaces}

One of the principal results in the theory of uniform spaces is the possibility of imbedding an arbitrary uniform space in a complete uniform space as a dense subspace. This problem has been treated by Bourbaki ([1], [2]) in two different ways. To the present author it seems that the situation becomes more lucid, if the construction of Bourbaki is modified by using a trick of Kowalsky ([3], p. 159). This modification yields the following, more precise result:

Theorem: For every uniform space $E$ there exists a complete uniform space $\tilde{E}$, containing $E$ as a dense subspace, and such that every point of $\tilde{E}-E$ is $T_{0}$-separated from every other point of $\tilde{E}$. Two complete spaces $\tilde{E}_{1}$ and $\tilde{E}_{2}$, satisfying these conditions, are always uniformly isomorphic and even in such a way that the identity mapping $E \rightarrow E$ has a unique extension to a uniform isomorphism $\tilde{E}_{1} \rightarrow \tilde{E}_{2}$.

Corollary: For every uniform $T_{0}$-space $E$ there exists a complete uniform $T_{0}$-space $\tilde{E}$, containing $E$ as a dense subspace. If $\tilde{E}_{1}$ and $\tilde{E}_{2}$ are two complete $T_{0}$-spaces, containing $E$ as a dense subspace, then the identity mapping $E \rightarrow E$ has a unique extension to a uniform isomorphism $\tilde{E}_{1} \rightarrow \tilde{E}_{2}$.

The purpose of this paper is to prove the theorem stated above and deduce the corollary from it.

If $(E, \mathfrak{w})$ is a uniform space, $\mathfrak{w}$ being its uniformity, we denote the uniform topology of $(E, \mathfrak{w})$ by $\tau_{\mathfrak{w}}$ and the neighborhood filter of a point $x$ of the topological space $\left(E, \tau_{\mathfrak{w}}\right)$ by $\tau_{\mathfrak{w}}(x)$. If $\mathfrak{u}$ is a filter of $E$ and $M$ a subset of $E$, we denote the trace of $\mathfrak{H}$ in $M$ by $\mathfrak{u}_{M}$. The induced uniformity of $M$ is, however, denoted by $\mathfrak{w}_{M}$ instead of $\mathfrak{w}_{\boldsymbol{M} \times \boldsymbol{M}}$. If $W$ is a set of $\mathfrak{w}$ and $x$ a point of $E$, the set of all points $y$ in $E$ such that $(x, y) \in W$ is denoted by $W[x]$.

1. Minimal Cauchy filters. A filter $\mathfrak{t}$ of a uniform space $(E, \mathfrak{w})$ is called open, if it has a base every set of which is open in the topology $\tau_{\mathfrak{w}}$. 
A. I. 333

It is called a Cauchy filter, if for every set $W$ of $\mathfrak{w}$ there exists a set $A$ of $\mathfrak{u}$ such that $A \times A \subset W$. If $\mathfrak{u}$ is a Cauchy filter of $(E, \mathfrak{w})$, then its trace $\mathfrak{H}_{M}$ in an arbitrary subspace $\left(M, \mathfrak{w}_{M}\right)$ is a Cauchy filter of this subspace.

A uniform space $(E, \mathfrak{w})$ is said to be complete, if every Cauchy filter of $(E, \mathfrak{w})$ is convergent in the uniform topology $\tau_{\mathfrak{w}}$, i.e. if it contains, as a subfamily, at least one neighborhood filter $\tau_{\mathfrak{w}}(x)$.

The family of all Cauchy filters of a uniform space $(E, \mathfrak{w})$ is partially ordered by inclusion. The maximal element of this partially ordered family is the filter that consists of all subsets of $E$, including the empty set $\varnothing$. We denote this filter by $\mathfrak{v}$ and call it the zero filter of $E$. The minimal elements of the partially ordered family of all Cauchy filters are called minimal Cauchy filters. In other words, a Cauchy filter $\mathfrak{U}$ of $(E, \mathfrak{w})$ is minimal, if from the relation $\mathfrak{v} \subset \mathfrak{u}$ it necessarily follows that the Cauchy filter $\mathfrak{v}$ is $=\mathfrak{u}$.

Every neighborhood filter $\tau_{w}(x)$ is a minimal Cauchy filter and every minimal Cauchy filter is open. Furthermore, for every Cauchy filter $\mathfrak{u} \neq 0$ there exists a unique minimal Cauchy filter $\mathfrak{v}$ such that $\mathfrak{v} \subset \mathfrak{u}$. It is readily seen that if $\mathfrak{a}$ and $\mathfrak{b}$ are two minimal Cauchy filters, such that $A \cap B \neq \varnothing$ for every $A$ in $\mathfrak{a}$ and every $B$ in $\mathfrak{b}$, then $\mathfrak{a}=\mathfrak{b}$. In fact, the family $\mathfrak{c}=\{A \cup B: A \in \mathfrak{a}, B \in \mathfrak{b}\}$ is then a Cauchy filter, satisfying $\mathfrak{c} \subset \mathfrak{a}$ and $\mathfrak{c} \subset \mathfrak{b}$, and from this it follows that $\mathfrak{a}=\mathfrak{c}=\mathfrak{b}$. Finally we observe that in a complete uniform space $(E, \mathfrak{w})$ the family of all minimal Cauchy filters coincides with the family of all neighborhood filters $\tau_{w}(x)$.

2. The construction of $\tilde{\boldsymbol{E}}$. Let $(E, \mathfrak{w})$ be an arbitrary uniform space. We denote by $\tilde{E}$ the set whose elements are

(a) the points of $E$,

(b) those minimal Cauchy filters of $(E, \mathfrak{w})$ that do not coincide with any neighborhood filter $\tau_{1 w}(x)$.

We define a mapping $\varphi$ of $E$ onto the family of all minimal Cauchy filters of $(E, \mathfrak{w})$ by setting $\varphi(x)=\tau_{\mathfrak{w}}(x)$ for every $x$ in $E$ and $\varphi(\tilde{x})=\tilde{x}$ for every $\tilde{x}$ in the class (b).

With every set $W$ of $\mathfrak{w}$ we associate the set $\tilde{W}^{r}$ consisting of all pairs $(\tilde{x}, \tilde{y})$, for which there exists a set $A \in q(\tilde{x}) \cap \psi(\tilde{y})$ such that $A \times A \subset W$. It is easy to see that

$$
\overline{V \cap W}=\tilde{V} \cap \tilde{W}
$$

for every pair $(V, W)$ of sets of $\mathfrak{w}$. From this it immediately follows that the family 


$$
\tilde{\mathfrak{W}}=\{\tilde{W}: W \in \mathfrak{w}\}
$$

is a base of a filter $\tilde{\mathfrak{w}}$ in $\tilde{E} \times \tilde{E}$. We will show that $\tilde{\mathfrak{w}}$ is a uniformity for $\tilde{E}$.

Since each $\varphi(\tilde{x})$ is a Cauchy filter, every pair $(\tilde{x}, \tilde{x})$ belongs to every set of the family $\tilde{\mathfrak{N}}$. Thus every set of $\tilde{\mathfrak{W}}$ contains the diagonal of $\tilde{E} \times \tilde{E}$.

From the definition of the sets $\tilde{W}$ it immediately follows that each of these sets is symmetric.

Finally, let $\tilde{W}$ be an arbitrary set of $\tilde{\mathfrak{S}}$, and let $V$ be a set of $\mathfrak{w}$ such that $V \circ V \subset W$. We consider an arbitrary element $(\tilde{x}, \tilde{y})$ of $\tilde{V} \circ \tilde{V}$. If $\tilde{z}$ is an element of $\tilde{E} \operatorname{such}$ that $(\tilde{x}, \tilde{z})$ and $(\tilde{z}, \tilde{y})$ belong to $\tilde{V}$, then there exists a set $A$ in $\varphi(\tilde{x}) \cap \varphi(\tilde{z})$ and a set $B$ in $\varphi(\tilde{z}) \cap \varphi(\tilde{y})$ such that $A \times A \subset V$ and $B \times B \subset V$. Since $A \cap B$ belongs to $\varphi(\tilde{z}) \neq \mathfrak{v}$, $A \cap B$ is non-empty. From this it readily follows that $A \times B \subset$ $V \circ V \subset W$ and, consequently,

$$
(A \cup B) \times(A \cup B) \subset W,
$$

if $W$ is symmetric. Since, on the other hand, $A \cup B$ belongs to $\varphi(\tilde{x}) \cap \varphi(\tilde{y})$, the pair $(\tilde{x}, \tilde{y})$ thus belongs to $\tilde{W}$, and we have

$$
\tilde{V} \circ \tilde{V} \subset \tilde{W} \text {. }
$$

The filter $\tilde{\mathfrak{w}}$ is therefore a uniformity for $\tilde{E}$.

3. $\boldsymbol{E}$ as a subspace of $\tilde{\boldsymbol{E}}$. We consider the trace $\tilde{\mathfrak{w}}_{E}$ of the uniformity $\tilde{\mathfrak{w}}$ in $E \times E$. Let $W$ be a set of $\mathfrak{w}$. If $(x, y)$ belongs to $\tilde{W} \cap(E \times E)$, then there exists a set $A$ in $\varphi(x) \cap \varphi(y)=\tau_{\mathfrak{w}}(x) \cap \tau_{\mathfrak{w}}(y)$ such that $A \times A \subset W$. The points $x$ and $y$ then belong to $A$ and, consequently, $(x, y)$ belongs to $A \times A \subset W$. Thus we have

$$
\tilde{W} \cap(E \times E) \subset W
$$

for every set $W$ in $\mathfrak{w}$.

Conversely, let $(x, y)$ be an arbitrary element of $W$. Since $\tau_{\mathfrak{w}}(x)$ and $\tau_{\mathfrak{w}}(y)$ are Cauchy filters, there exists a set $A$ in $\tau_{\mathfrak{w}}(x)$ and a set $B$ in $\tau_{\mathfrak{w}}(y)$ such that $A \times A \subset W$ and $B \times B \subset W$. For an arbitrary element $(u, v)$ of $A \times B$ we then get $(u, x) \in A \times A \subset W,(x, y) \in W$ and $(y, v) \in B \times B \subset W$ and, consequently, $(u, v) \in W^{3}$. We thus see that $A \times B \subset W^{3}$ and therefore

$$
(A \cup B) \times(A \cup B) \subset W^{3},
$$

$\mathbf{i}_{\mathrm{f}} W$ is symmetric. Since $A \cup B \in \tau_{\mathfrak{w}}(x) \cap \tau_{\mathfrak{w}}(y)$, the pair $(x, y)$ therefore belongs to $\widetilde{W^{3}}$. This indicates that 


$$
W \subset \widetilde{W^{3}} \cap(E \times E)
$$

for every symmetric set $W$ in $\mathfrak{w}$. From the relations (1) and (2) it finally follows that $\tilde{\mathfrak{w}}_{E}=\mathfrak{w}$, and $(E, \mathfrak{w})$ is thus a subspace of $(\tilde{E}, \tilde{\mathfrak{w}})$.

Now we show that $E$ is dense in $\left(\tilde{E}, \tau_{\tilde{\mathfrak{w}}}\right)$. In order to do this we consider an arbitrary symmetric set $W$ of $\mathfrak{w}$ and an arbitrary point $\tilde{x}$ of $\tilde{E}$. Since $\varphi(\tilde{x})$ is a Cauchy filter of $(E, \mathfrak{w})$, there exists a set $A$ of this filter satisfying $A \times A \subset W$. Since $\tilde{x}$ is not the zero filter, the set $A$ is nonempty. Let $a$ be a point of $A$. From the Cauchy filter $\varphi(a)=\tau_{w}(a)$ we choose a set $B$ such that $B \times B \subset W$. For an arbitrary element $(u, v)$ of $A \times B$ we then get $(u, a) \in A \times A \subset W$ and $(a, v) \in B \times B \subset W$ and, consequently, $(u, v) \in W^{2}$. From this it follows, as before, that

$$
(A \cup B) \times(A \cup B) \subset W^{2} .
$$

Since $A \cup B \in \varphi(\tilde{x}) \cap \varphi(a)$, the pair $(\tilde{x}, a)$ thus belongs to $\widetilde{W^{2}}$. Therefore we have

$$
\widetilde{W^{2}}[\tilde{x}] \cap E \neq \varnothing,
$$

which shows that $E$ is dense in $(\tilde{E}, \tilde{\mathfrak{w}})$.

4. The completeness of $\tilde{E}$. In this section we first prove the following lemma, which will primarily be used in section 6 , but which will also to some extent shorten the proof of the completeness of $\tilde{E}$ :

Lemma: If $M$ is a dense subspace of a uniform space $E$, then $\mathfrak{a} \rightarrow \mathfrak{a}_{M}$ is a one-to-one mapping from the family of all minimal Cauchy filters of $E$ onto the family of all minimal Cauchy filters of $M$.

Let first $\mathfrak{a}$ be a minimal Cauchy filter of $E$. Then $\mathfrak{a}_{M}$ is a Cauchy filter in $M$ and there exists, consequently, a minimal Cauchy filter $\mathfrak{H}$ of $M$ for which $\mathfrak{i t} \subset \mathfrak{a}_{M}$. Let $\mathfrak{i t}$ be the filter of $E$, with $\mathfrak{u}$ as a base. If $A$ were a set of $\mathfrak{a}$, such that $A \cap M$ would not belong to $\mathfrak{u}$, then $A$ could not belong to $\overrightarrow{\mathfrak{H}}$, and thus $\overline{\mathfrak{H}} \cap \mathfrak{a}$ were a Cauchy filter of $E$ properly contained in the minimal Cauchy filter $\mathfrak{a}$. This contradiction shows that $\mathfrak{u}=\mathfrak{a}_{M}$, and thus the trace in $M$ of every minimal Cauchy filter of $E$ is a minimal Cauchy filter of $M$.

Conversely, let $\mathfrak{u}$ be a minimal Cauchy filter of $M$. This filter $\mathfrak{u}$ is a base of a Cauchy filter $\overline{\mathfrak{t}}$ in $E$. Let $\mathfrak{v}$ be a minimal Cauchy filter of $E$ such that $\mathfrak{v} \subset \mathfrak{\mathfrak { H }}$. Then we have $\mathfrak{v}_{M} \subset \overline{\mathfrak{H}}_{M}=\mathfrak{H}$ and therefore $\mathfrak{v}_{M}=\mathfrak{u}$, since $\mathfrak{v}_{M}$ is a Cauchy filter and $\mathfrak{u}$ is minimal. Thus every minimal Cauchy filter of $M$ is the trace of at least one minimal Cauchy filter of $E$. 
At last we see that if $\mathfrak{a}$ and $\mathfrak{b}$ are two minimal Cauchy filters of $E$ such that $\mathfrak{a}_{M}=\mathfrak{b}_{M}(\neq \mathfrak{D}$ since $M$ is dense), then every set of $\mathfrak{a}$ intersects every set of $\mathfrak{b}$ and, consequently, $\mathfrak{a}=\mathfrak{b}$. Our lemma is proved.

In order to prove that $(\tilde{E}, \tilde{\mathfrak{w}})$ is complete, we consider an arbitrary nonzero Cauchy filter $\tilde{\mathfrak{u}}$ of $(\tilde{E}, \tilde{\mathfrak{w}})$. We have to show that there exists at least one point $\tilde{x}$ of $\tilde{E}$ such that $\tau_{\tilde{\mathfrak{w}}}(\tilde{x}) \subset \tilde{\mathfrak{u}}$. Since there exists a minimal Cauchy filter $\tilde{\mathfrak{v}}$ satisfying $\tilde{\mathfrak{v}} \subset \tilde{\mathfrak{u}}$, we may assume that $\tilde{\mathfrak{u}}$ is a minimal Cauchy filter. From our lemma it then follows that the trace $\tilde{\mathfrak{H}}_{\boldsymbol{E}}$ of $\tilde{\mathfrak{u}}$ is a minimal Cauchy filter of $(E, \mathfrak{w})$. Let $\tilde{x}$ be a point of $\tilde{E}$ such that $\varphi(\tilde{x})=\tilde{\mathfrak{l}}_{E}$. We show that

$$
\tilde{\mathfrak{u}}=\tau_{\tilde{\mathfrak{w}}}(\tilde{x}) .
$$

Since $\tilde{\mathfrak{H}}$ and $\tau_{\tilde{\mathfrak{w}}}(\tilde{x})$ are minimal Cauchy filters, the assertion is proved, if we show that every set of $\tilde{\mathfrak{l}}$ intersects every set of $\tau_{\tilde{\mathfrak{w}}}(\tilde{x})$. To see this we consider an arbitrary set $\tilde{A}$ of $\tilde{\mathfrak{i}}$ and an arbitrary set $W$ of $\mathfrak{w}$. Let $V$ and $U$ be symmetric sets of $\mathfrak{w}$ such that $V^{2} \subset W$ and $U^{3} \subset V$. Since $E$ is dense in $(\tilde{E}, \tilde{\mathfrak{w}})$, we can choose a point $x$ from $\tilde{U}[\tilde{x}] \cap E$. From the relation $(\tilde{x}, x) \in \tilde{U}$ it follows that there exists a set $B$ in $\varphi(\tilde{x}) \cap \varphi(x)=$ $\tilde{\mathfrak{H}}_{E} \cap \tau_{\mathfrak{w}}(x)$ satisfying $B \times B \subset U$. Since both $\tilde{A} \cap E$ and $B$ belong to the non-zero filter $\tilde{\mathfrak{u}}_{E}$, their intersection $\tilde{A} \cap B$ is non-empty. If $y$ is a point of $\tilde{A} \cap B$, then by (2)

$$
(x, y) \in B \times B \subset U \subset \widetilde{U^{3}} \subset \tilde{V} .
$$

From this and from $(\tilde{x}, x) \in \tilde{U} \subset \tilde{V}$ it then follows that

$$
(\tilde{x}, y) \in \tilde{V} \circ \tilde{V} \subset \tilde{W}
$$

i.e. that $y \in \tilde{W}[\tilde{x}]$. This shows that the intersection $\tilde{W}[\tilde{x}] \cap \tilde{A}$ is nonvoid and thus the equation (3) holds. From this equation it finally follows that $\tilde{\mathfrak{l}}$ converges to $\tilde{x}$, and thus $(\tilde{E}, \tilde{\mathfrak{w}})$ is complete.

5. The postulate $T_{0}$. Let $(\tilde{x}, \tilde{y})$ be an element of $\tilde{E} \times \tilde{E}$ belonging to every set $\tilde{W}$. For every set $W$ of $\mathfrak{w}$ it then exists a set $M$ of $\varphi(\tilde{x}) \cap \varphi(\tilde{y})$ satisfying $M \times M \subset W$. This shows that the family

$$
\mathfrak{c}=\{A \cup B: A \in \varphi(\tilde{x}), B \in \varphi(\tilde{y})\}
$$

is a Cauchy filter in $(E, \mathfrak{w})$. Since it is included in both $\varphi(\tilde{x})$ and $\varphi(\tilde{y})$, we have thus $\varphi(\tilde{x})=\mathfrak{c}=\varphi(\tilde{y})$. Therefore we see that if $(\tilde{x}, \tilde{y})$ is a pair of elements of $\tilde{E}$ for which $\varphi(\tilde{x}) \neq \varphi(\tilde{y})$, then there always exists a set $\tilde{W}$ of 
$\tilde{\mathfrak{w}}$ such that $(\tilde{x}, \tilde{y}) \notin \tilde{W}$. The set $\tilde{W}[\tilde{x}]$ is then a neighborhood of $\tilde{x}$ that does not contain $\tilde{y}$. This indicates that every point of $\tilde{E}-E$ is $T_{1^{-}}$ separated from every other point of $\tilde{E}$.

6. The uniqueness of the completion. Let now $(\tilde{E}, \tilde{\mathfrak{w}})$ and $(\hat{E}, \hat{\mathfrak{w}})$ be two completions of $(E, \mathfrak{w})$ satisfying the conditions of our theorem. Let $\tilde{x}$ be an arbitrary point of $\tilde{E}$. Then the trace $\tau_{\tilde{\mathfrak{w}}}(\tilde{x})_{E}$ of $\tau_{\tilde{\mathfrak{w}}}(\tilde{x})$ is, according to the lemma of section 4 , a minimal Cauchy filter of $(E, \mathfrak{m})$. After this, the same lemma shows that $\tau_{\tilde{\mathfrak{w}}}(\tilde{x})_{E}$ is the trace in $E$ of a minimal Cauchy filter $\hat{\mathfrak{u}}$ of $(\hat{E}, \hat{\mathfrak{w}})$. Since $(\hat{E}, \hat{\mathfrak{w}})$ is complete, there exists a point $\hat{x}$ of $\hat{E}$ such that $\hat{\mathfrak{u}}=\tau_{\hat{\mathfrak{w}}}(\hat{x})$. Thus we see that with every point $\tilde{x}$ of $\tilde{E}$ we can associate a point $\hat{x}$ of $\hat{E}$ in such a way that

$$
\tau_{\tilde{\mathfrak{w}}}(\tilde{x})_{E}=\tau_{\widehat{\mathfrak{w}}}(\hat{x})_{E} .
$$

If, in particular, $\tilde{x}$ lies in $E$, we may obviously suppose that $\hat{x}=\tilde{x}$. Now we consider the mapping $f:(\tilde{E}, \tilde{\mathfrak{w}}) \rightarrow(\hat{E}, \hat{\mathfrak{w}})$ defined by

$$
f(\tilde{x})=\hat{x} .
$$

If $\tilde{x}$ is in $\tilde{E}-E$, then $\hat{x}$ is in $\hat{E}-E$. In fact, if $\hat{x}$ were in $E$, then

$$
\tau_{\tilde{\mathfrak{w}}}(\tilde{x})_{E}=\tau_{\hat{\mathfrak{w}}}(\hat{x})_{E}=\tau_{\mathfrak{w}}(\hat{x})_{E}
$$

and, consequently, $\tau_{\tilde{\mathfrak{w}}}(\tilde{x})=\tau_{\tilde{\mathfrak{w}}}(\hat{x})$. This is a contradiction, however, since the points of $\tilde{E}-E$ are $T_{1}$-separated from other points of $(\tilde{E}, \tilde{\mathfrak{w}})$. If $\hat{x}$ is an arbitrary point of $\hat{E}-E$, then $\tau_{\hat{1 \mathfrak{v}}}(\hat{x})_{E}$ is a minimal Cauchy filter of $(E, \mathfrak{w})$ and, as a consequence, there exists a point $\tilde{x}$ of $\tilde{E}$ such that

$$
\tau_{\tilde{\mathfrak{w}}}(\tilde{x})_{E}=\tau_{\hat{\mathfrak{w}}}(\hat{x})_{E} .
$$

By (4) we then have $\tau_{\hat{\mathfrak{w}}}(f(\tilde{x}))=\tau_{\hat{\mathfrak{w}}}(\hat{x})$, i.e. $f(\tilde{x})=\hat{x}$. Since $f$ is the identity in $E, \tilde{x}$ belongs to $\tilde{E}-E$. This shows that $f$ is a mapping from $\tilde{E}-E$ onto $\hat{E}-E$. Finally we observe that if $f(\tilde{x})=f(\tilde{y})$, then

$$
\tau_{\tilde{\mathfrak{w}}}(\tilde{x})_{E}=\tau_{\hat{\mathfrak{w}}}(f(\tilde{x}))_{E}=\tau_{\hat{\mathfrak{w}}}(f(\tilde{y}))_{E}=\tau_{\tilde{\mathfrak{w}}}(\tilde{y})_{E}
$$

and, consequently, $\tau_{\tilde{\mathfrak{v}}}(\tilde{x})=\tau_{\tilde{\mathfrak{w}}}(\tilde{y})$. From this it follows, if $\tilde{x}$ and $\tilde{y}$ belong to $\tilde{E}-E$, that $\tilde{x}=\tilde{y}$. This indicates that $f$ is a one-to-one mapping from $\tilde{E}-E$ onto $\hat{E}-E$. Since it coincides in $E$ with the identity mapping, it is thus a one-to-one map from $\tilde{E}$ onto $\hat{E}$.

Next we prove that $f$ is a uniform isomorphism. Because of the symmetry it suffices to show that $f$ is uniformly continuous. Let $\hat{W}$ be an 
arbitrary symmetric set of $\hat{\mathfrak{w}}$. Since the restriction $f \mid E:(E, \mathfrak{w}) \rightarrow(\hat{E}, \hat{\mathfrak{w}})$ is uniformly continuous, there exists a set $\tilde{W}$ in $\tilde{\mathfrak{w}}$ such that

$$
\tilde{W}^{3} \cap(E \times E) \subset \hat{W} .
$$

We consider an arbitrary point $(\tilde{x}, \tilde{y})$ of $\tilde{W}$. From (4) it follows that there exists a symmetric set $\tilde{V}$ of $\tilde{\mathfrak{w}}$ such that $\tilde{V} \subset \tilde{W}$ and

$$
\tilde{V}[\tilde{x}] \cap E \subset \hat{W}[f(\tilde{x})] \cap E, \tilde{V}[\tilde{y}] \cap E \subset \hat{W}[f(\tilde{y})] \cap E .
$$

Since $E$ is dense in $\tilde{E}$, we can choose a point $x$ from $\tilde{V}[\tilde{x}] \cap E$ and a point $y$ from $\tilde{V}[\tilde{y}] \cap E$. From the relations $(x, \tilde{x}),(\tilde{y}, y) \in \tilde{V} \subset \tilde{W}$ and $(\tilde{x}, \tilde{y}) \in \tilde{W}$ it follows that $(x, y) \in \tilde{W}^{3}$ and therefore, by $(5)$, that $(x, y) \in \hat{W}$. On the other hand, by $(6) x \in \hat{W}[f(\tilde{x})]$ and $y \in \hat{W}[f(\tilde{y})]$, i.e. $(f(\tilde{x}), x) \in \hat{W}$ and $(y, f(\tilde{y})) \in \hat{W}$ and, consequently, $(f(\tilde{x}), f(\tilde{y})) \in \hat{W}^{3}$. Thus we see that from $(\tilde{x}, \tilde{y}) \in \tilde{W}$ it always follows that $(f(\tilde{x}), f(\tilde{y})) \in \hat{W}^{3}$. This proves that $f$ is a uniform isomorphism, as we wished to show.

We have seen that the identity mapping $i:(E, \mathfrak{w}) \rightarrow(E, \mathfrak{w})$ can be extended to a uniform isomorphism $f:(\tilde{E}, \tilde{\mathfrak{w}}) \rightarrow(\hat{E}, \hat{\mathfrak{w}})$. It remains to be shown that this extension is unique. In order to see this, we suppose that $g:(\tilde{E}, \tilde{\mathfrak{w}}) \rightarrow(\hat{E}, \hat{\mathfrak{w}})$ is another such extension and that $f(\tilde{x}) \neq g(\tilde{x})$. Then the points $f(\tilde{x})$ and $g(\tilde{x})$ belong to $\hat{E}-E$ and, as a consequence, $\tau_{\hat{\mathfrak{w}}}(f(\tilde{x}))$ $\neq \tau_{\hat{\mathfrak{w}}}(g(\tilde{x}))$. From this it follows, since the neighborhood filters are minimal Cauchy filters, that there exist disjoint sets $\hat{A} \in \tau_{\hat{w}}(f(\tilde{x}))$ and $\hat{B} \in \tau_{\hat{w}}(g(\tilde{x}))$. On the other hand, we see that, since $f$ and $g$ are continuous, there exists a neighborhood $\tilde{U}$ of $\tilde{x}$ such that $f(\tilde{U}) \subset \hat{A}$ and $g(\tilde{U}) \subset \hat{B}$. Since $E$ is dense in $\tilde{E}$, we can choose a point $x$ from $\tilde{U} \cap E$. Then the point $x=f(x)=g(x)$ belongs to $f(\tilde{U}) \cap g(\tilde{U})$ and, consequently, to $\hat{A} \cap \hat{B}$. This contradiction shows that $f$ is unique.

7. Proof of the corollary. Now we suppose that $(E, \mathfrak{w})$ is a uniform $T_{0}$-space and show that then $(\tilde{E}, \tilde{\mathfrak{w}})$ is a $T_{0}$-space, too. We have seen above that every point of $\tilde{E}-E$ is $T_{0}$-separated from every other point of $\tilde{E}$. Thus we only have to consider two points $x$ and $y$, both belonging to $E$. If they are not $T_{0}$-separated, then $\tau_{\tilde{w}}(x)=\tau_{\tilde{w}}(y)$ and, consequently, $\tau_{\tilde{\mathfrak{w}}}(x)_{E}=\tau_{\tilde{\mathfrak{w}}}(y)_{E}$. On the other hand, since both $\tau_{\tilde{\mathfrak{w}}}(x)_{E}$ and $\tau_{\mathfrak{w}}(x)$ are minimal Cauchy filters in $(E, \mathfrak{w})$ and since every set of the former filter intersects every set of the latter filter (at $x)$, we see that $\tau_{\mathfrak{w}}(x)_{E}=\tau_{\mathfrak{w}}(x)$ and, likewise, $\tau_{\mathfrak{w}}(y)_{E}=\tau_{\mathfrak{w}}(y)$. Thus $\tau_{\mathfrak{w}}(x)=\tau_{\mathfrak{w}}(y)$, from which it finally follows, since $(E, \mathfrak{w})$ is a $T_{0}$-space, that $x=y$. This completes the proof of the corollary. 


\section{References}

[1] Bourbaki, N.: Topologie générale, Chap. II, second edition, Paris 1951.

[2] -»- Topologie générale, Chap. II, third edition, Paris 1961.

[3] Kowalsky, H.-J.: Topologische Räume, Basel und Stuttgart 1961.

Printed March 1963. 